\title{
Classified Measurement of Household Load for Smart Meter
}

\author{
Yaolun $\mathrm{Xu}^{1}$, Yuansheng Gao ${ }^{1}$, Ping Dai ${ }^{1}$, Jinhui Zou ${ }^{1}$ and Yun Gao ${ }^{2}$ \\ ${ }^{1}$ Dazhou Electricity Industry Bureay Dazhou, Sichuan Province, China \\ ${ }^{2}$ School of Electrical and Information University of Sichuan Chengdu, Sichuan Province, China \\ gaoyun.scu.88.03@163.com
}

\begin{abstract}
The classified measurement of household load can make home users keep abreast of the electricity consumption in their home and guide them use electricity reasonably, and at the same time strengthen the demand side management (DSM). Load identification is the base of the classified measurement of household load. According to the shortcomings of the existing load identification which often use the steady state characteristics as the signature, and considering the uniqueness of the transient characteristics of household load, a method was proposed to recognize the time it turns on or off, and then measure the electricity of each load based on Minkowski distance used transient waveform of the active power and reactive power as the signature. Firstly, set up a template library as the comparative standard to recognize. And then calculate the matching degree between the waveform acquired and in template library to determine at the moments which load turns on or off or no load changes its state. Afterwards the time each load used is obtained. Finally, on the basis of the use of time of each load, calculate its power consumption. The process of the method is simple, and the result by measured data has proved the method has high accuracy and feasibility. It can be used for classified measurement of household load for smart meter.

Index Terms - Non-intrusive, smart meter, classified measurement, Minkowski measure, matching degree.
\end{abstract}

\section{Introduction}

Demand side management (DSM) is required to guide users the rationality of electricity usage, improve the terminal efficiency ${ }^{[1,2]}$. Only allow the users to understand their electricity consumption timely can enable them to respond positively to DSM ${ }^{[3]}$, and then realize the interaction on electricity with electric utilities ${ }^{[4]}$. Smart meters need to provide technical support for DSM ${ }^{[2]}$, because they are the important infrastructure for smart grid construction ${ }^{[5]}$. The smart meters used now only have the capability of timesharing metering the electricity. They don't have the function of classified measurement of load, and don't provide enough real-time information for electric users and utilities ${ }^{[6]}$. For the reason to make the users join the interaction and possible arrange the electricity using time reasonably, accurate load identification and classified measurement of the load according to the overall power consumption are needed ${ }^{[7]}$.

The main monitoring method existing for measurement by classification of the load is intrusive and non-intrusive ${ }^{[8]}$. Intrusive load monitoring installs instrument on each load interested for separate measure ${ }^{[9]}$, and it's expensive ${ }^{[7]}$. Nonintrusive load monitoring only need to install the meter on the incoming cables, it's low cost and suitable to online monitoring ${ }^{[10]}$. It is a obvious trend that the intrusive load monitoring is supplanted by the non-intrusive monitoring for its advantages ${ }^{[11]}$, and the latter is the principal method of monitoring. However the electricity consumption is complex and diversified and its electrical characteristics have some connection with the operating environment, and then the key point of the load identification and measurement by classification is how to pick out the useful data. In order to solve this problem, the researchers proposed some method, such as edge detection, clustering analysis, artificial neural nets (ANN) and so on ${ }^{[12]}$. They all can identify the main household load in a certain error range, but they don't measure the electricity by classification.

A method to solve the question of classified measurement is proposed. It has three steps: data preprocessing, matching and identification, and classified measurement. Firstly, determine the matching sub-library by analyzing the changing trend of the acquired data. Secondly, calculate the trend matching degree and partial matching degree by Minkowski distance, and then determine which load turns on or off, or no changes happen on the time, based on the maximum matching degree. Finally, calculate the electricity consumption of each class of the load according to the time it turns on and off. The function of the classified measurement is achieved. The method and process is simple, and accurate. It can be used for the smart meter to finish the capacity of classified measurement.

\section{Transient Waveform}

There are many signatures of the load, mainly including transient state and steady state characteristics ${ }^{[13]}$. Transient state process includes more useful information than steady state, and easier to identify, because the transient state characteristics have a close relationship with their physical properties and missions. So the transient state characteristics are used as signatures to identify the load in this paper. The active power transient waveforms of the household load classes monitored are showed in table 1. It shows that the differences among the different classes of the load are huge. There are two columns in the table including opening process and closing process.

The trend of the load when it's opening or closing is clearly. For example, when the load is opening, the active power must increase after short-run changes, and when 
closing, it must decrease. So it can be roughly divided into two sub-libraries by analyzing the trend changes.

TABLE I Transit Waveform

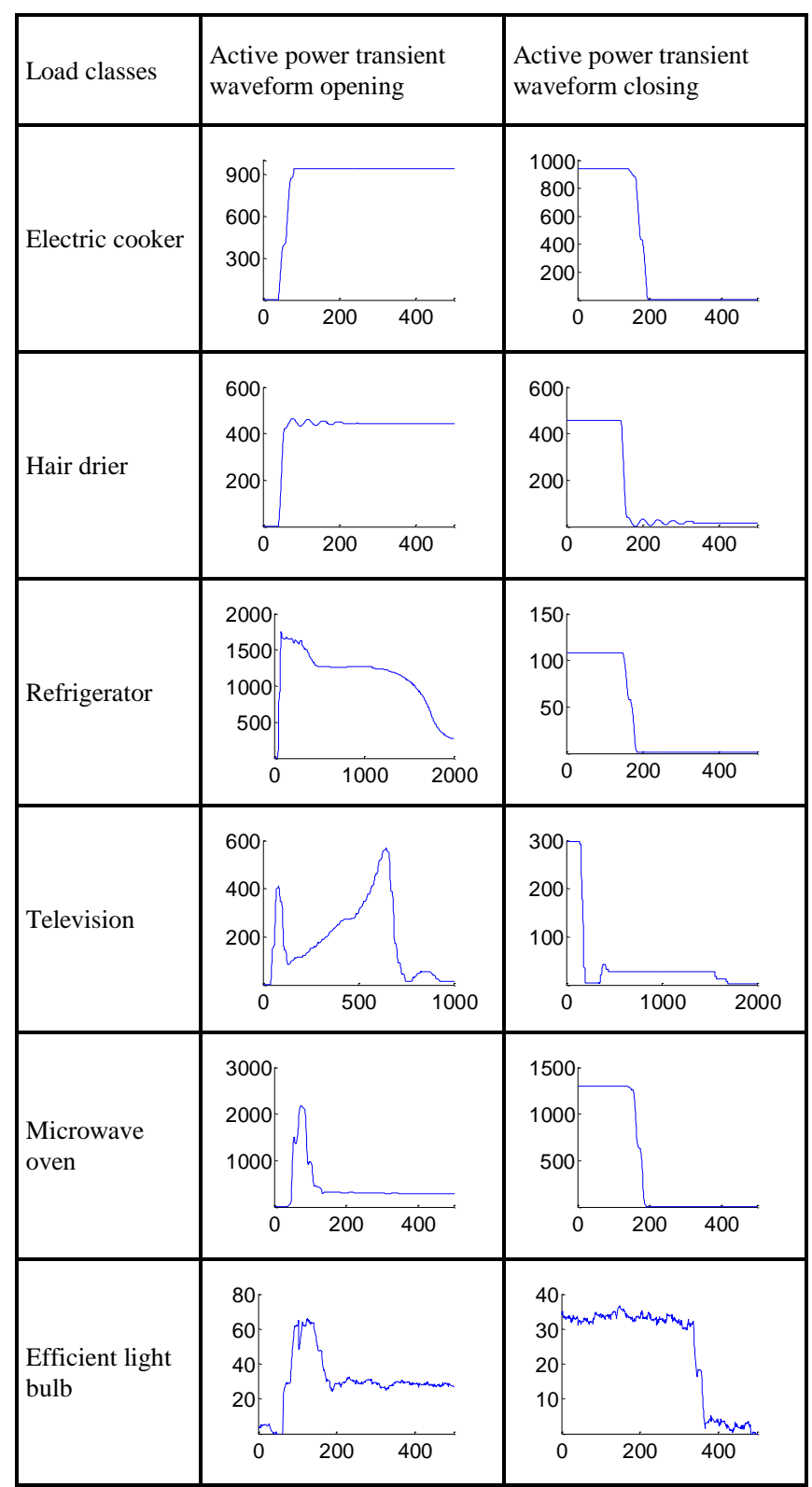

Note: horizontal axis: time, vertical axis: active power and reactive power

\section{Minkowski Distance}

Minkowski distance is a space measuring method. The Minkowski distance between two $n$-d vectors $\boldsymbol{x}\left(x_{1}, x_{2}, \ldots, x_{n}\right)$, $\boldsymbol{y}\left(y_{1}, y_{2}, \ldots, y_{n}\right)$ is defined as:

$$
\operatorname{dist}(\boldsymbol{x}, \boldsymbol{y})=\left(\frac{1}{n} \sum_{i=1}^{n}\left|x_{i}-y_{i}\right|^{p}\right)^{1 / p}
$$

The $p$ in the deification can take a positive integer, $\operatorname{dist}(\boldsymbol{x}, \boldsymbol{y}) \in[0, \infty)$. Then the matching degree can be expressed as:

$$
\gamma(\boldsymbol{x}, \boldsymbol{y})=\frac{1}{1+\operatorname{dist}(\boldsymbol{x}, \boldsymbol{y})}
$$

$$
1+\operatorname{dist}(\mathrm{x}, \mathrm{y}) \in[1, \infty), \gamma(\boldsymbol{x}, \boldsymbol{y}) \in(0,1] \text {. }
$$

Household load transient waveform sequence can be seen as an $n$-dimensional vector. The degree of difference or matching can be measured by Minkowski distance. From above, when $\operatorname{dist}(\boldsymbol{x}, \boldsymbol{y})$ is more closely to $0, \gamma(\boldsymbol{x}, \boldsymbol{y})$ is more closely to 1 , the degree of difference between them is smaller and the matching degree is greater, conversely, the degree of difference is greater and matching degree is smaller.

\section{Application in Household Load Measurement by Classification}

Accurate identification of household load is the premise of classified measurement in non-intrusive monitoring conditions. The way to identify the load needs some differences in the characteristics. And so use the easily recognizable transient waveform of the load as the signature to identify. The process is showed in figure 1 .

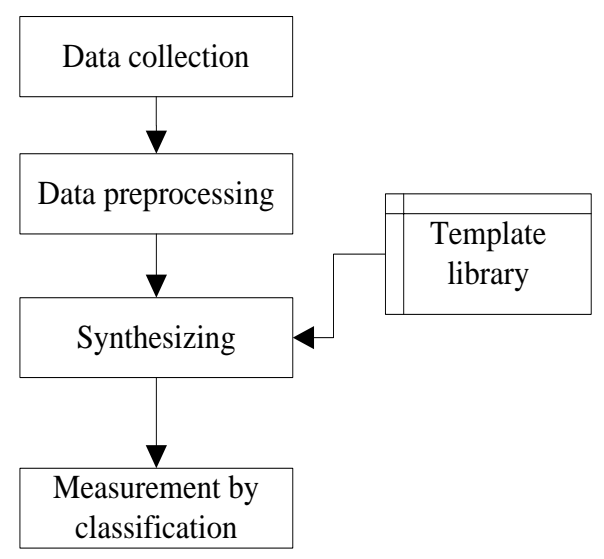

Fig.1 Diagram of load recognition process

\section{A. Household Load Template Library}

The knowledge of the load characteristics and the differences between them should have before the identification. Establishing the template library is the premise of accurate identification.

Repeat the measurement of the household load establishing the template library to avoid the random fluctuation of the household electricity and power supply quality. Measure each load alone in a continuous period, and then count the statistical waveform of the transient characteristics in the library. The template library includes steady active power, active power statistical waveform and reactive power statistical waveform in transient process, and the partial interval of these waveforms.

The library is separated into two parts to reduce the search range, opening sub-library and closing sub-library stores the template data of opening and closing process separately. 


\section{B. Data Preprocessing}

It would produce large error if take the transient waveform collected as the signature directly. One of the reasons is that there is a certain degree of interference in the data collection. It affects the calculation of Minkowski distance. On the other hand, in order to highlight the characteristics of the mutation part of the transient waveform better, the partial waveform is extracted, and compared with the others' separately. So the step is needed, it can weaken the noise, and get the trend changes, reduce the matching range.

\section{Synthesize the Matching Results}

This step is critical. It synthesizes the global and partial characteristics of the active power and reactive power of household load.

\section{1) Calculate Global and Partial Matching Degree}

According to the trend changes calculate the global matching degree of the samples collected and the templates in the library by Minkowski distance. Based on the partial characteristics exacted calculate the partial matching degree by Minkowski distance.

The calculations of the global matching degree and partial matching degree are paralleled process, so it can save some time.

\section{2 ) Synthesize the Identifying Results}

Synthesize the global matching degree and partial matching degree to get the estimation of the matching degree. Give consideration to the affections to the household load identification of the both by weighted average.

$$
\hat{\boldsymbol{g}}=\alpha \boldsymbol{\gamma}_{g l o}+(1-\alpha) \boldsymbol{\gamma}_{\text {sub }}
$$

In the formulation, $\hat{\boldsymbol{g}}=\left[\begin{array}{llll}\hat{g}_{1} & \hat{g}_{2} & \cdots & \hat{g}_{n}\end{array}\right]$ is the estimation of the matching degree, $\gamma_{g l o}$ is the global matching degree, and $\gamma_{\text {sub }}$ is the partial matching degree, $\alpha$ is the weight, $n$ is the number of the templates in the library, $0 \leq \hat{g}_{i} \leq 1, i=1,2, \ldots, n$.

Take the templates in the library as a test set to obtain the optimal weight. The objective function is the sum of squares of the differences between estimating and actual matching degree, it is formulated in (4). In the formulation, $\boldsymbol{g}$ is the actual matching degree. The estimating matching degree $\hat{\boldsymbol{g}}$ is related to the parameter $p$ of the Minkowski distance and the weight $\alpha$. So the F is the function of $p$ and $\alpha$. In the condition of meeting the formulation (5), the $\alpha$ which can minimize the formulation (4) is the optimal weight.

$$
F(p, \alpha)=\|\boldsymbol{g}-\hat{\boldsymbol{g}}\|^{2}
$$

$$
\alpha<1
$$

It is a least-squares problem with conditions, and solves the problem to obtain the optimal weight $\alpha$ The results are listed in the table 2. Table 2 shows the time used with different weights and Minkowski distance parameter $p$.
TABLE II Values of Weights, Results of Objective Function and Time Used

\begin{tabular}{|c|c|c|c|}
\hline$p$ & Weight & Objective function value & Time/(ms) \\
\hline 1 & 0.5734 & 0.0623 & 3.1268 \\
\hline 2 & 0.8027 & 0.0131 & 2.4731 \\
\hline 3 & 0.8631 & 0.0113 & 13.8217 \\
\hline 4 & 0.8918 & 0.0200 & 13.8223 \\
\hline 5 & 0.9100 & 0.0158 & 13.8206 \\
\hline 6 & 0.9225 & 0.0288 & 13.8236 \\
\hline
\end{tabular}

The errors are both small when $p=2 、 3$, but the time used when $p=2$ is six times of the time used when $p=3$. So choose $p=2$ and $\alpha=0.8027$ to participate in the calculation of the load identification.

In load identification, take a weighted average of the global and partial matching degree to obtain the synthesizing matching degree. Choose the max synthesizing matching degree as the results.

\section{Classified Measurement of Electricity Consumption}

According to the results obtained by the steps abovementioned, record the time of the load opening and closing. The electricity consumption of each load is obtained by multiplying the active power value stored in the library and the time difference between opening and closing, showed in formulation (6). The formulation (7) is the error.

$$
\begin{aligned}
& \hat{W}=P \Delta t \\
& \varepsilon(\%)=\frac{\hat{W}-W}{W} \times 100
\end{aligned}
$$

In the formulation, $W$ is the actual electricity consumption, $\hat{W}$ is the estimating electricity consumption, $P$ is the active power value stored in the template library, is the time difference between opening and closing.

\section{Test by Real Data}

It's a non-intrusive load monitoring because the monitoring meter was installed on the incoming cables. On the same time, monitor the interesting load individually, and record the time and the electricity consumption as a standard for comparing. The details are showed in table 3 .

TABLE III Testing Conditions

\begin{tabular}{|l|l|l|}
\hline \multirow{4}{*}{$\begin{array}{l}\text { Monitoring } \\
\text { conditions }\end{array}$} & Time & $14^{\text {th }}$ May $-26^{\text {th }}$ May \\
\cline { 2 - 3 } & Place & Chengdu \\
\cline { 2 - 3 } & Meter & DL750 \\
\cline { 2 - 3 } & Location & $\begin{array}{l}\text { Incoming cables of the home; } \\
\text { Incoming cables of each interesting load }\end{array}$ \\
\cline { 2 - 3 } & $\begin{array}{l}\text { Sampling } \\
\text { frequency }\end{array}$ & $2 \mathrm{kHz}$ \\
\hline
\end{tabular}


Set the parameters according to the results in 4.3.2: $p=2$, $\alpha=0.8027$. The accuracy of the load identification is $95 \%$.

\section{A. Results of Classified Measurement of Electricity Consumption}

In the above monitoring conditions, the details of electricity use in the home are obtained. Take the data of a workday on $24^{\text {th }}$ May as an example to confirm the accuracy. Figure 2 shows the total active power changes with time. The electricity use is mainly concentrated in 7:00-8:30 and 18:0024:00.

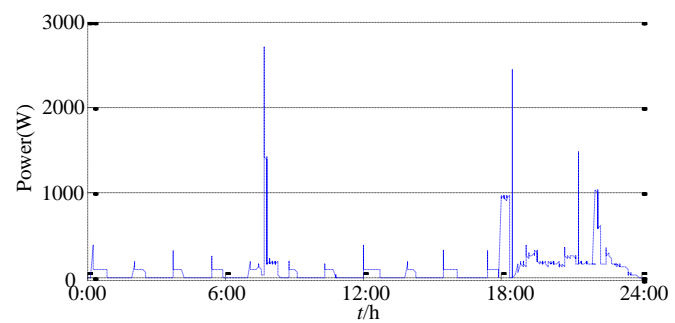

Fig. 2 The active power of the home monitored on a workday

Identify the opening and closing times by the data showed in figure 2 as the steps in 4.3, and obtain the time division of the total electricity use into each load, and showed in figure 3. It agrees with the results monitored individually.
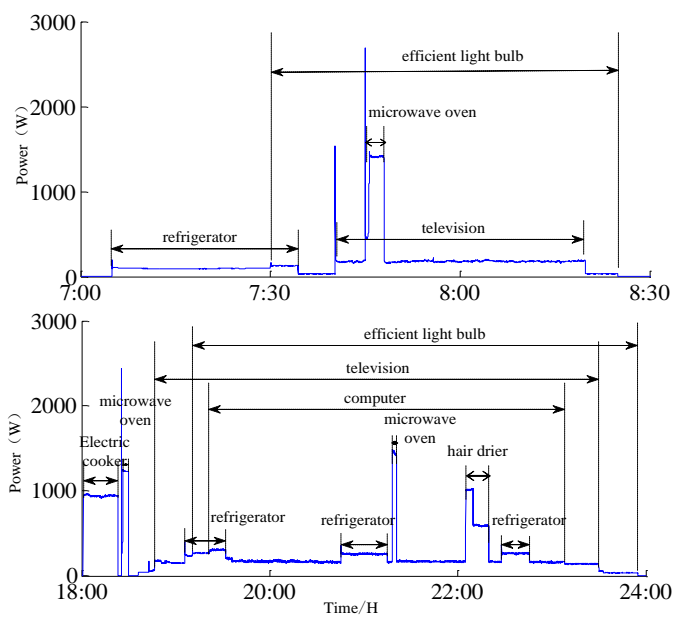

Fig. 3 The time division diagram of power consumption on morning and evening
Calculate the electricity consumption of each load according to the time it used by the formulation (6). The figure 4 shows the each load electricity consumption occupying the percentage of total electricity consumption. The electricity consumption is concentrated on the refrigerator and television. They occupy the total electricity more than $50 \%$.

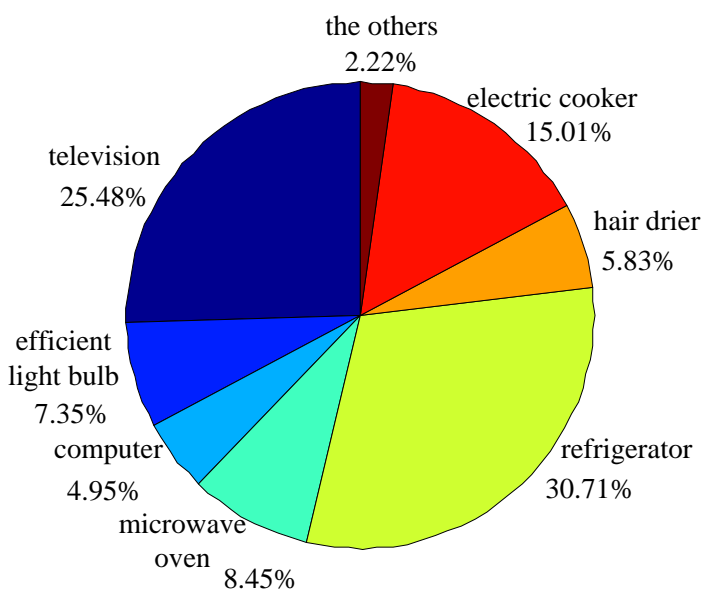

Fig.4 The percentage of power consumption of each household load

\section{B. Error Analysis}

The error between the estimated and actual electricity consumption is showed in figure 5. The error comes from the step of identification and the measurement. In the step of identifying the load opening and closing time, one hand, the classes of the load may be wrong, on the other hand, the time may have errors. But the identifying time is to one second for the benchmark. The affects of the time error to the measurement is minimal and are disregarded. The measurement error is mainly due to the active power of the stored value in the library is a statistical value. The error is unavoidable for the load which active power has large fluctuations. The following results confirmed the analysis of the error.

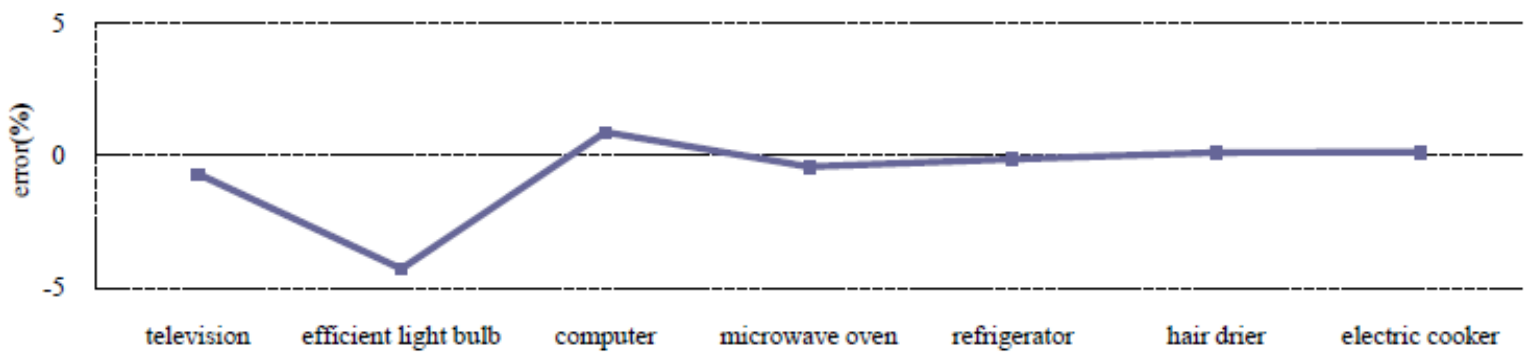

Fig. 5 The error between the estimated and actual power consumption of each appliance 
From the figure above, the estimated errors of the television, computer, and efficient light bulb are larger than the others. Take the actual use time and electricity consumption for a comparison, it showed that the error of time is small, but the fluctuation percentage of the active power value is larger than others'. The main reason of the large error is that the difference between actual active power average value and the value stored in the template library is bigger.

\section{Conclusion}

The classified measurement of household load can optimize the power consumption mode of the home, and make the users take participation in DSM. Measure the load by classification with synthesizing the global and partial characteristics by Minkowski distance. It takes a more completely use of time domain characteristics of transient waveform. The method is simple and to implement, and the error of the classified measurement of the common load in home is smaller than $5 \%$, so it can be used for the smart meter.

But the estimated error exists and is large, as the actual active power of some load has large fluctuations, and has a large difference with the value stored in the library. The next step of the research is how to store the active power value to make it more close to the real satiation.

\section{References}

[1] Beibei Wang and Yang Li, "Demand side management planning and implementation mechanism for smart grid," Electric Power Automation Equipment, vol. 30, no. 12, pp. 19-24, December 2010.

[2] Beibei Wang, Yang Li and Ciwei Gao. "Demand Side Management Outlook under Smart Grid Infrastructure," Automation of Electric Power Systems, vol. 33, no. 22, pp. 17-22, October 2009.
[3] Tiantian Liang, Ciwei Gao and Beibei Wang, "Applications of demand side management in smart grid," Electric Power Automation Equipment, vol. 32, no. 4, pp. 81-85, May 2012.

[4] Luhua Zhang, Sitong Wang, Zhonglin Yi, Ruiming Yuan, Hui Zhou, Qingduo Yin, "The design and implementation of family comprehensive energy management system facing the smart power," Electrical Measurement and Instrumentation, vol. 47, no. 9, pp. 35-38, September 2010.

[5] Wei,Zhao Kang Yao and Songling Huang, "Uderstanding and recognition of the advanced metering infrastructure and its related concepts," Electrical Measurement and Instrumentation, vol. 47, no. 7, pp. 1-4, July 2010.

[6] Liang Chen, Fangcheng Lü, Qing Xie, Xingni Lan and Shaojun Sun, "Research of real-time energy measurement and bidirectional communication smart meter," Proceedings of the CSEE, vol. 31, pp. 9499, December 2011.

[7] Peng Li, Non-intrusive power load disaggregation and monitoring, Tianjin, CA: Tianjin University, 2009.

[8] George W. Hart, "Nonintrusive appliance load monitoring," Proceedings of the IEEE, vol. 80, no. 12, pp. 1870-1891, December 1992,.

[9] Chunliu Zhao, A study on the applications of the steady state harmonics based non-intrusive power load disaggregation method, Tianjin, CA: Tianjin University, 2009

[10] Lulu Niu and Hongjie Jia, "Transient event detection algorithm for nonintrusive load monitoring," Automation of Electric Power Systems, vol. 35, no. 9, pp. 30-35, May 2011.

[11] K.Suzuki, S.Inagaki, T. Suzuki, H.Nakamura and K. Ito, "Nonintrusive appliance load monitoring based on integer programming," SICE Annual Conference, Tokyo, Japan, pp. 2742-2747, August 2008.

[12] Arend J. Biker, Xiaohua Xia and Jiangfeng Zhang, "Active power residential non-intrusive appliance load monitoring system," IEEE Conferences, Africon, pp. 1-6, September 2009.

[13 ]H.Najmeddine, et al., "State of Art on load monitoring methods," 2nd IEEE International Power and Energy Conference, Johor Bahru, Malaysia, pp. 1256-1258, December 2008. 